\title{
Utilizing Forster Resonance Energy Transfer to Extend Spectral Response of PCDTBT:PCBM Solar Cells
}

\author{
Erik Klump ${ }^{\mathrm{a}}$, Iordania Constantinou ${ }^{\mathrm{b}}$, Tzung-Han Lai ${ }^{\mathrm{a}}$, Franky So $^{\mathrm{b}}$ \\ ${ }^{a}$ Department of Materials Science and Engineering, University of Florida, Gainesville, FL 32611, \\ United States \\ ${ }^{b}$ Department of Materials Science and Engineering, North Carolina State University, Raleigh, \\ NC 27607, United States \\ Corresponding Author: Franky So \\ Email address: fso@ncsu.edu
}




\begin{abstract}
Light harvesting in the near-infrared part of the solar spectrum is important to achieve high efficiency polymer solar cells (PSCs). In this work, we demonstrate that we take an existing polymer:fullerene blend and extend its spectral response into the near-IR region by adding a small amount near-IR absorbing dye in the blend. The polymer studied in this work is Poly[ $N-9^{\prime}-$ heptadecanyl-2,7-carbazole-alt-5,5-(4',7'-di-2-thienyl-2',1',3'-benzothiadiazole)] (PCDTBT). By adding only $2.5 \%$ squaraine dye to the blend, we were able to extend the spectral response of the resulting devices $100 \mathrm{~nm}$ into the near-IR spectral region. We show that the enhanced light harvesting is due to efficient Forster resonance energy transfer (FRET) between PCDTBT and the squaraine dye, resulting in an increase in power conversion efficiency. This type of ternary polymer solar cells is unique in that it allows the use of a small amount of selected dyes to extend light harvesting in infrared region.
\end{abstract}

\title{
Graphical Abstract
}
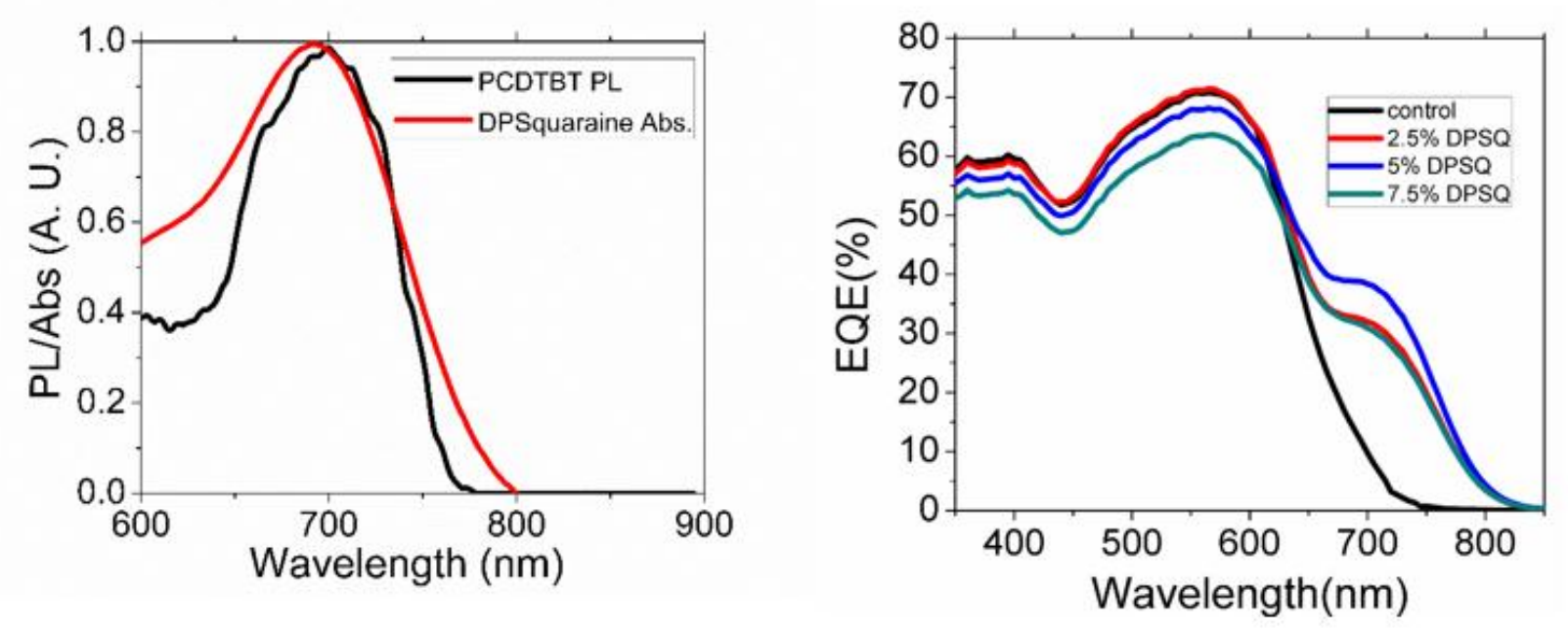


\section{Introduction}

Polymer solar cells (PSCs) have generated significant attention as a possible low-cost alternative to conventional inorganic crystalline cells due in part to their compatibility with rollto-roll processing [1-3]. One present challenge for PSCs is to increase their utilization of solar irradiation by extending their spectral response into the near-IR region. Poly[ $N-9^{\prime}$-heptadecanyl2,7-carbazole-alt-5,5-(4',7'-di-2-thienyl-2',1',3'-benzothiadiazole)] (PCDTBT) is a photovoltaic polymer that has been demonstrated to give respectable solar cells with power conversion efficiencies of 5-7\% [4, 5] with a spectral cutoff at about $700 \mathrm{~nm}$, leaving much of the solar spectrum unused. This challenge has been tackled by design and synthesis of lower bandgap polymers absorbing longer wavelength light, [2, 6-8] but PSCs with these polymers typically have to sacrifice open-circuit voltage $\left(\mathrm{V}_{\mathrm{OC}}\right)$ to realize the extended spectral response, thus negating the benefits of low bandgaps. Devices using a tandem structure have also been explored wherein each subcell covers a different spectral region [9-11], but optimizing the interlayers and matching the photocurrent generated in each cell is another challenge.

Recently, various types of ternary blend PSCs have been explored including those utilizing a large fraction of a third absorber to extend the spectral response [12-14]. However, maintaining the necessary morphology for efficient charge collection has proven to be another challenge, as a large fraction of a third component, typically over $20 \%$ by weight, is required to facilitate efficient charge transport. Another strategy to extend light harvesting is to incorporate a small amount of dyes and other sensitizers in the photoactive layer to avoid the morphology issue associated with a third component in a blend. In order for these dyes to be an efficient sensitizer, they must possess a very high absorption coefficient so as to efficiently absorb the long wavelength light with only a small concentration in the photoactive layer. Additionally, 
there must be efficient energy and/or charge transfer from the dye to the primary components in order to harvest excitons generated by the dye. Previous studies on these dye doped devices have focused on enhancing the spectral response of P3HT/PCBM devices [15-19]. However, P3HT has a relatively large bandgap with an absorption edge at $650 \mathrm{~nm}$. Demonstrating this strategy using a lower bandgap polymer is highly desirable.

One promising family of molecules that work well as a sensitizer in PSCs are squaraines. Because of their high absorption coefficients, different squaraine molecules have been used in different photovoltaic applications as a sole donor [20, 21], ternary blends with other small molecule donors and PCBM [22, 23], and dye doped blends with P3HT and PCBM [15, 18, 24]. Previous work focused on the P3HT/PCBM system where the spectral response was extended to around $720 \mathrm{~nm}$ using squaraine [15]. To ensure efficient light harvesting with a small amount of squaraine, Forster resonance energy transfer (FRET) must be efficient between the polymer and the dye dopant. FRET is a long range energy transfer process (on the order of several nanometers) due to dipole-dipole interaction between the donor and the acceptor molecules [25]. The likelihood for this energy transfer process to occur is strongly dependent on the overlap between the donor's emission spectrum and the acceptor's absorption spectrum. This relationship is shown in the following expression:

$$
R_{0} \propto\left[\int F_{D}(\lambda) \varepsilon_{A}(\lambda) \lambda^{4} d \lambda\right]^{1 / 6}
$$

where $\mathrm{R}_{\mathrm{o}}$ is the Forster radius which is the distance between donor and acceptor molecules where FRET is $50 \%$ efficient, $\mathrm{F}_{\mathrm{D}}$ is the normalized emission spectrum of the donor molecule, $\varepsilon_{A}$ is the molar extinction coefficient of the acceptor molecule, and $\lambda$ is the wavelength of light. OSC's have utilized FRET in a variety of ways beyond use in dye doped cells including small molecule 
bilayer cells [26] and polymer ternary blend BHJ cells [27]. FRET facilitates the electronic coupling between the donor polymer and the acceptor dye and has been shown to enhance exciton harvesting $[3,15]$. Because of the low dye concentration used in sensitized OSCs to extend the spectral response to longer wavelengths [15], the effects on the polymer: fullerene blend morphology are minimized.

In this work, we demonstrate that a sensitizer can be used to extend the absorption wavelength by $100 \mathrm{~nm}$ in a PSC with PCDTBT:[6,6]-phenyl C71 butyric acid methyl ester $\left(\mathrm{PC}_{71} \mathrm{BM}\right)$ as a photoactive layer. The dye used here is 2,4-Bis[4-( $N, N$-diphenylamino)-2,6 dihydroxyphenyl] squaraine (DPSQ). In these cells, FRET is efficient between DPSQ and PCDTBT and the presence of the dye has no negative effect on the external quantum efficiency (EQE) of the original cell. We confirmed that FRET occurred by observing strong quenching of the photoluminescence of PCDTBT due to the presence of DPSQ, even when the materials were separated by a spacer layer. This resulting dye doped cell has an expanded spectral response to $800 \mathrm{~nm}$ corresponding to the DPSQ absorption, with an increase in short circuit current contributing to a $10 \%$ increase in PCE. This approach was found to be successful at low concentrations of DPSQ; however, the carrier lifetime, the mobility and hence the device performance deteriorate as the dye concentration increases.

\section{Experimental}

Materials. PCDTBT, PC71BM and DPSQ were purchased from 1-Material, Nano-C and Sigma Aldrich, respectively, and used without further purification.

Device Fabrication. PCDTBT and PCBM in a ratio of 1:4 were dissolved in dichlorobenzene at a concentration of $20 \mathrm{mg} / \mathrm{mL}$. Various concentrations of DPSQ were also 
dissolved into this solution. Pre-patterned ITO substrates were cleaned via sonication in acetone and isopropanol and 10 minutes of UV-ozone treatment. Poly(3,4-ethylenedioxythiophene) polystyrene sulfonate (PEDOT:PSS) was spin-coated onto the ITO substrates at $8000 \mathrm{rpm}$ and heated to $140^{\circ} \mathrm{C}$ for 20 minutes. The PCDTBT:PC 71 BM:DPSQ solution was then spin-coated on top of the ITO substrates at a rate of $2000 \mathrm{rpm}$. Substrates were then transferred in a high vacuum thermal evaporator where lithium fluoride and aluminum were deposited to form the top electrode.

Device Characterization. A tungsten filament lamp along with a monochromator was used as a light source for external quantum efficiency measurements. The monochromatic light was calibrated using a silicon detector. A Stanford Research Systems SRS 830 lock-in amplifier was used for lock-in detection at a chopping frequency of $270 \mathrm{~Hz}$. Current density-voltage (J-V) measurements were carried out under AM $1.5 \mathrm{G}$ irradiation at $100 \mathrm{~mW} \mathrm{~cm}^{-2}$.

\section{Results and Discussion}

The energy level alignment of the dye-doped polymer solar cell system is depicted in Figure 1a. The highest occupied molecular orbital (HOMO) energy of DPSQ is 5.3 eV [28] and aligns well with the HOMO energy of PCDTBT which is important to allow holes to move freely between DPSQ and the polymer. The lowest unoccupied molecular orbital (LUMO) energy of DPSQ is $3.8 \mathrm{eV}$ resulting in a small LUMO-LUMO offset between DPSQ and PC ${ }_{71} \mathrm{BM}$ to facilitate efficient exciton dissociation at the DPSQ/PC71BM interface.

To determine whether the dye is efficient for FRET, we measured the absorption spectrum of DPSQ and the photoluminescence spectrum of PCDTBT, and the data are shown in Figure $1 \mathrm{~b}$. The absorption of DPSQ was found to start at roughly $650 \mathrm{~nm}$ with a peak at 
approximately $700 \mathrm{~nm}$ and an absorption coefficient about three times that of PCDTBT [25], and its absorption maximum $(690 \mathrm{~nm})$ is almost the same as the PL maximum $(700 \mathrm{~nm})$ of PCDTBT, indicating FRET from PCDTBT to DPSQ should be possible. Since the typical Forster radius is on the order of a few nanometers for materials with good spectral overlap [15], so as long as the domain size is small in PCDTBT/DPSQ blend films, FRET should be efficient in devices fabricated with these materials in the active layer.
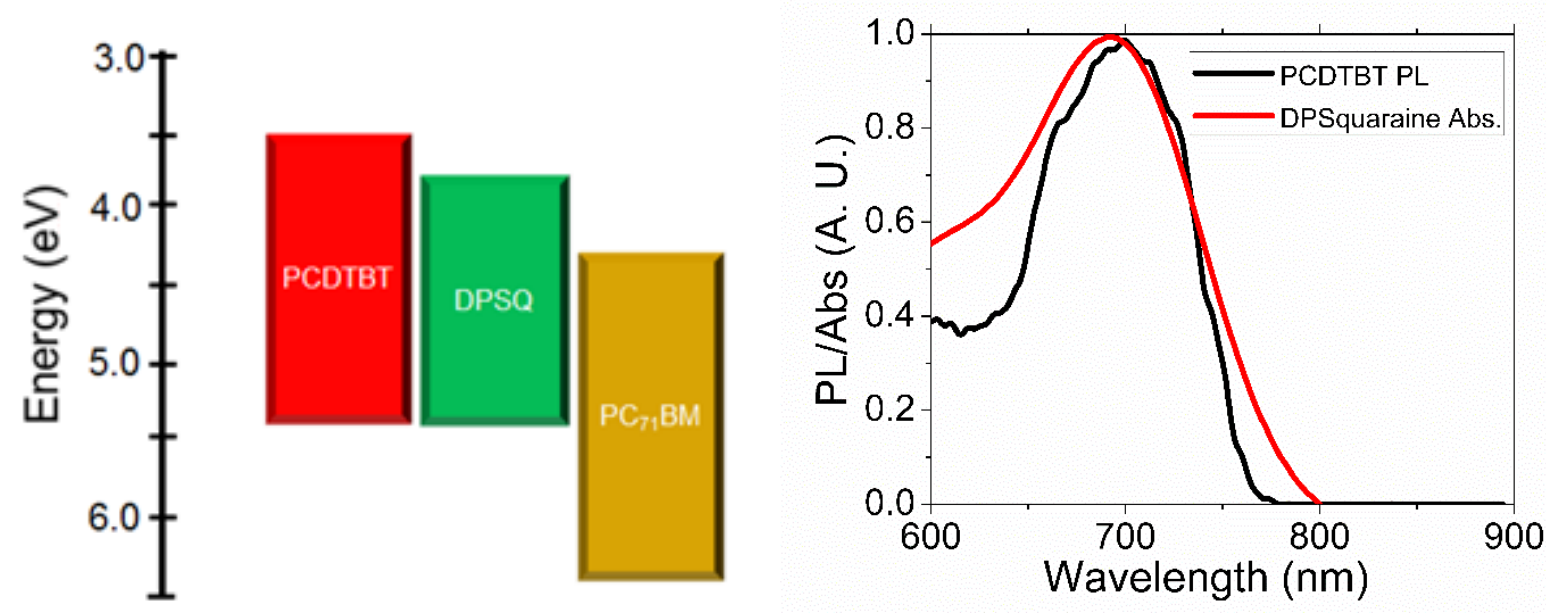

Fig. 1 (a) Energy level diagram for the PCDTBT/PC ${ }_{71}$ BM/DPSQ dye doped system. (a) normalized photoluminescence spectrum of PCDTBT and absorption spectrum of DPSQ;

In order to verify experimentally that FRET does in fact occur between PCDTBT and DPSQ, PL measurements were done on the PCDTBT,PCDTBT /DPSQ (20 nm) bilayer, and PCDTBT:DPSQ blend ( $5 \%$ by weight) samples using an excitation wavelength of $525 \mathrm{~nm}$. Here, the bilayer sample was prepared by evaporating a $20 \mathrm{~nm}$ thick layer of DPSQ on top of the PCDTBT film. As shown in Figure 2a, the photoluminescence signal can be detected between 650 and $750 \mathrm{~nm}$. When the DPSQ layer is present, this luminescence is quenched by almost 
$60 \%$. To confirm that the luminescence did not come from DPSQ, photoluminescence measurements were also done on pristine DPSQ and no PL signal was detected from DPSQ with an excitation at $525 \mathrm{~nm}$, indicating that the PL signal must be coming from PCDTBT only, indicating that the energy transfer between PCDTBT and DPSQ was efficient.

To further verify that the energy transfer is by FRET, a $7 \mathrm{~nm}$ thick layer of BCP was deposited onto the polymer by thermal evaporation prior to deposition of the DPSQ layer. When the photoluminescence of these samples was measured, PL was quenched by about $50 \%$, indicating that quenching is reduced with the presence of the BCP layer. As shown in Figure 2b, BCP's HOMO of $6.5 \mathrm{eV}$ and LUMO of $2.9 \mathrm{eV}$ [29] precludes the possibility of excitons diffusing through the BCP layer and into the DPSQ layer and exciton dissociation at the PCDTBT/BCP interface. Therefore, the demonstrated luminescence quenching is due to FRET of excitons from PCDTBT directly to DPSQ through the BCP spacer.
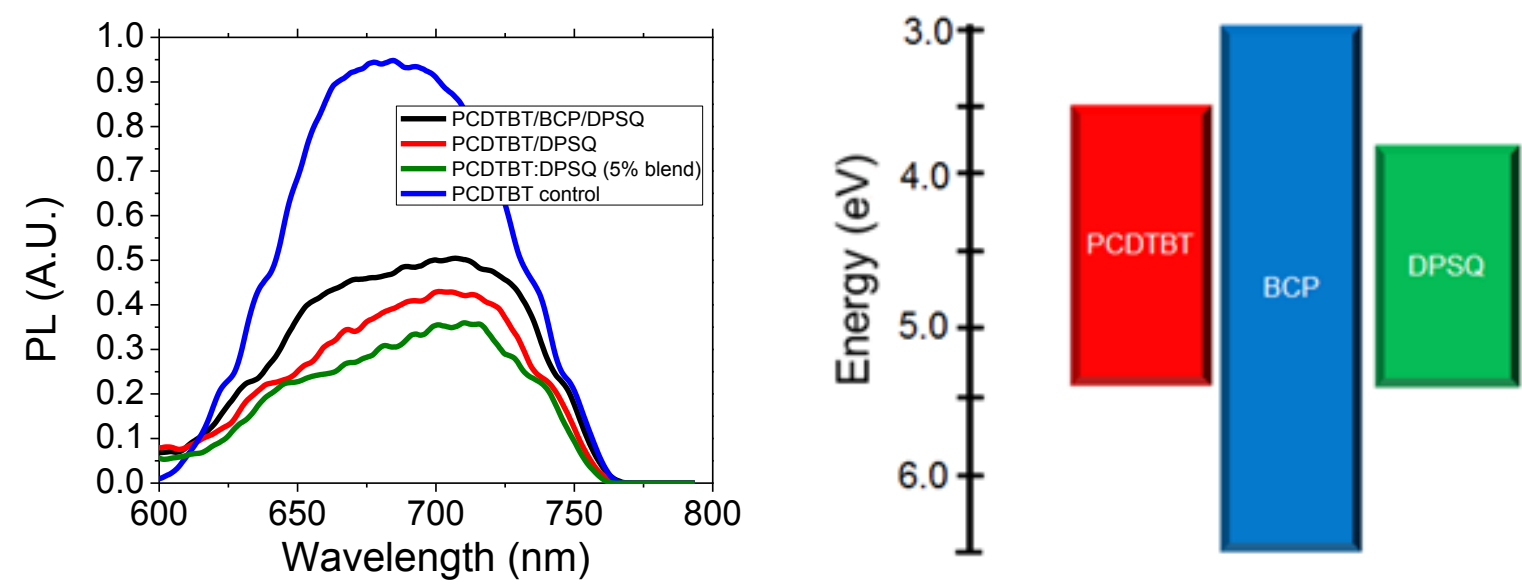

Fig. 2 a) Photoluminescence data from PCDTBT, PCDTBT/DPSQ, PCDTBT:DPSQ blend, and PCDTBT/BCP/DPSQ samples. b) Energy level alignment of photoluminescence samples. 
The photovoltaic performance of devices with DPSQ was investigated. The device structure used for this study was ITO/PEDOT:PSS/PCDTBT:PC 71 BM:DPSQ/LiF/Al. The concentration of DPSQ was varied $(0 \%, 2.5 \%, 5 \%$, and $7.5 \%$ by weight $)$ to investigate the effect of DPSQ on device performance. The current-voltage characteristics of devices under 1 sun illumination are shown in Figure 3a. These data show that as the concentration of DPSQ is varied, the $\mathrm{V}_{\mathrm{OC}}$ did not significantly change $(0.89 \mathrm{~V}$ to $0.87 \mathrm{~V})$. This behavior is consistent with DPSQ functioning as a sensitizer even at a concentration of $7.5 \%$ rather than as an organic alloy [30]. Consequently, we conclude that practically most carrier transport occurs via the PCDTBT and $\mathrm{PC}_{71} \mathrm{BM}$ phases rather than via DPSQ. The control device without DPSQ has a short-circuit current of $\left(\mathrm{J}_{\mathrm{SC}}\right) 10.1( \pm 0.30) \mathrm{mA} \mathrm{cm}^{-2}$, a fill factor of $58( \pm 1.5) \%( \pm 1.5)$ and a Voc of 0.89 $( \pm 0.01) \mathrm{V}$, resulting in a power conversion efficiency (PCE) of $5.2( \pm 0.2) \%$. At a DPSQ concentration of $2.5 \%$, the $\mathrm{J}_{\mathrm{SC}}$ significantly increases by $13 \%$ to $11.4( \pm 0.3) \mathrm{mA} \mathrm{cm}{ }^{-2}$ with a nearly identical fill factor of $57( \pm 2.1) \%$ and a Voc of $0.89( \pm 0.01) \mathrm{V}$, resulting in a PCE to 5.8 $( \pm 0.2) \%$. When the concentration of DPSQ is further increased to $5 \%, \mathrm{~J}_{\mathrm{SC}}$ decreases slightly to $11.1( \pm 0.22) \mathrm{mA} \mathrm{cm}^{-2}$ and the fill factor decreases significantly to $50( \pm 1.6) \%$. These effects result in a decrease in PCE to $5.1( \pm 0.16) \%$, roughly the same as the control device. This trend continues as the concentration of DPSQ is increased to $7.5 \%$ as the $\mathrm{J}_{\mathrm{SC}}$ decreases further to 10.9 $( \pm 0.25) \mathrm{mA} \mathrm{cm}^{-2}$, the fill factor decreases to $45( \pm 1.7) \%$, and PCE decreases to $4.3( \pm 0.2) \%$.

To further elucidate the photovoltaic performance of the dye doped blend devices, the external quantum efficiencies (EQEs) of the devices were measured. The resulting EQE spectra are depicted in Figure 3b. The EQE spectrum of the device with 2.5\% DPSQ shows that most of the increase in $\mathrm{J}_{\mathrm{SC}}$ is a result of the increased EQE from $650-800 \mathrm{~nm}$. This is the region where DPSQ absorbs strongly, resulting in a significant increase in EQE of $30 \%$ at $700 \mathrm{~nm}$ with a 
concentration of only $2.5 \%$ DPSQ in the dye doped blend devices. These results indicate that in the $2.5 \%$ DPSQ devices, the internal quantum efficiency increases significantly as a result of FRET facilitating more efficient exciton harvesting in the longer wavelengths due to the absorption of the dye. From the device results, we therefore conclude that at $2.5 \%$ concentration of DPSQ, FRET appears to be mitigating the morphological problems that are typical present in dye doped polymer-fullerene blend PSCs. As the DPSQ concentration further increases to 5\%, we see the long-wavelength EQE continues to increase to $37 \%$. However, this increase from the DPSQ dominated spectral region is offset by a slight decrease in EQE in the region contributed by PCDTBT. Once the DPSQ concentration increases to 7.5\%, the EQE decreases across all wavelengths, thereby leading to the observed decrease in $\mathrm{J}_{\mathrm{SC}}$.
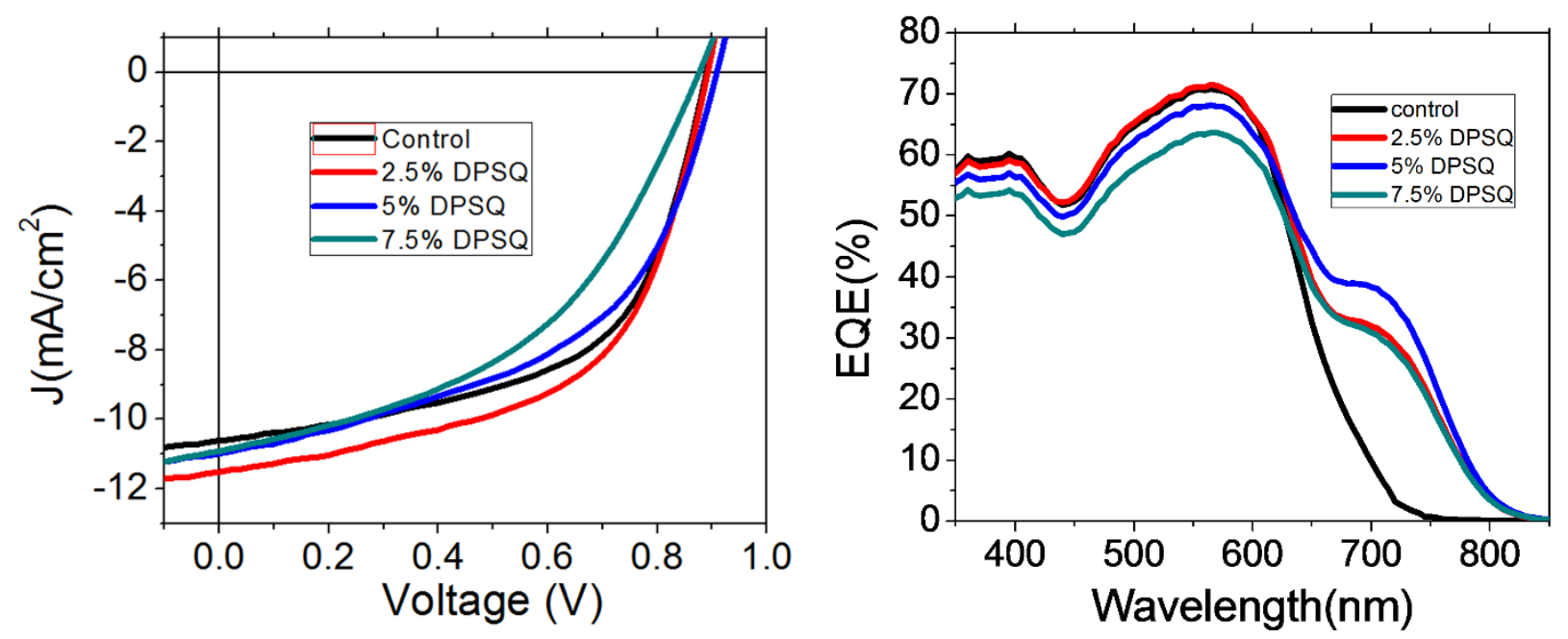

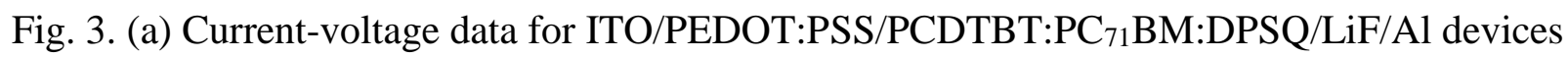
with varying DPSQ concentration; (b) EQE spectra for completed devices with varying DPSQ concentration. 
To verify the effect of FRET on the device performance, double-pass, reflective device transmission was measured and the data were used to calculate the device absorption using aluminum as the reflectance standard. These spectra are depicted in Figure 4a. Using these data, internal quantum efficiencies (IQEs) for different devices were determined and the results are shown in Figure 4b. At concentrations of 2.5\% and 5\% DPSQ, a modest increase in the short wavelength IQE is observed. As this is a region in which PCDTBT is the primary absorbing material rather than DPSQ, it is clear that FRET of excitons from PCDTBT to DPSQ helps increase exciton dissociation. Additionally, in the region beyond $700 \mathrm{~nm}$ wherein DPSQ absorbs strongly, an IQE of close to $90 \%$ is achieved. However, when the concentration of DPSQ is increased further to $10 \%$, the IQE drops across the entire spectrum with the most dramatic decrease occurring in the long wavelength region associated with DPSQ.
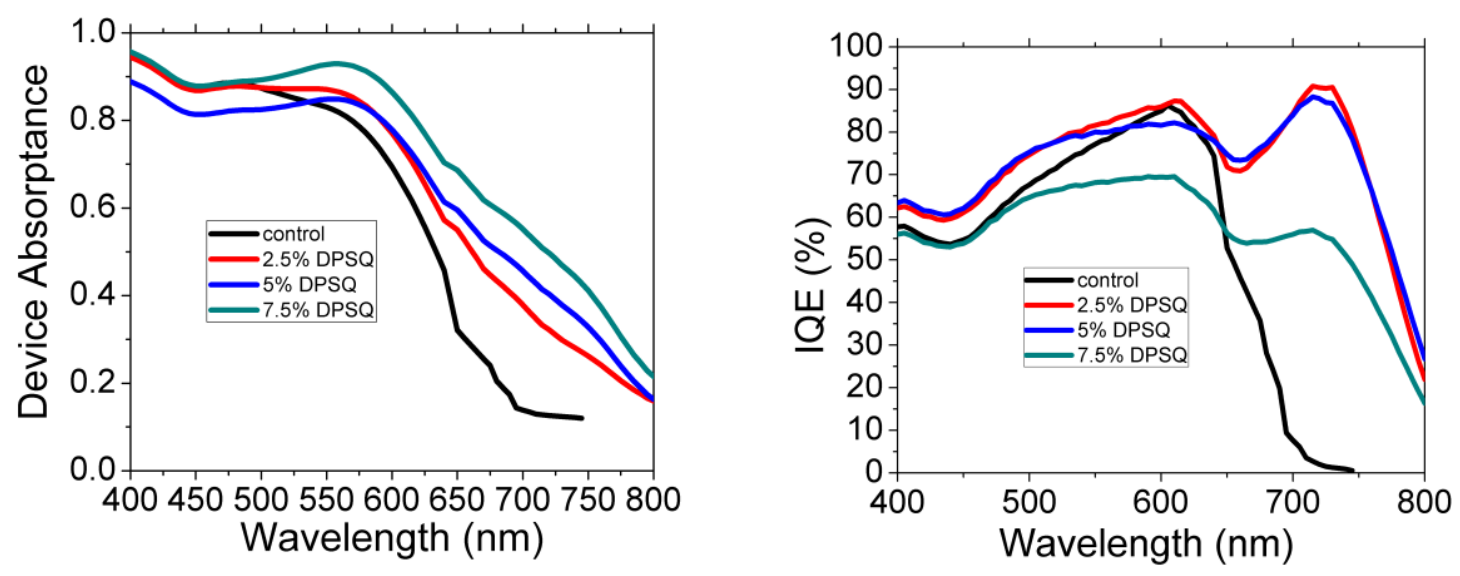

Fig. 4. (a) Double-pass, reflective absorptance spectra for devices with varying DPSQ concentration; (b) IQE spectra calculated from EQE and device absorptance for devices with varying DPSQ concentration

To probe the carrier dynamics of our dye doped blend devices, transient photovoltage (TPV) measurements were done on these devices, and the normalized data are shown in Figure 5. In TPV, a $527 \mathrm{~nm}$ laser was used to excite the devices held at open circuit conditions. The 
measured lifetime for the control device was $540 \mathrm{~ns}$. This lifetime decreased to $350 \mathrm{~ns}$ for the device with 2.5\% DPSQ. A further decrease to $330 \mathrm{~ns}$ occurred as the DPSQ concentration was increased to 5\%. This carrier lifetime trend is consistent with the observed decrease in fill factor. We conclude that this decrease in lifetime contributes to a decrease in fill factor, but is likely not significant enough to entirely account for the change in fill factor given the relatively small lifetime shift compared to the significant fill factor change. The electron mobility of the blend films were also measured via space charge limited current (SCLC) to further explain this fill factor decrease. In samples with the control active layer blend, the electron mobility was found to be $2.1 \times 10^{-4} \mathrm{~cm}^{2} \mathrm{~V}^{-1} \mathrm{~s}^{-1}$. This lifetime was reduced once $2.5 \%$ DPSQ was added, decreasing to $1.3 \times 10^{-4} \mathrm{~cm}^{2} \mathrm{~V}^{-1} \mathrm{~s}^{-1}$. Upon increasing the DPSQ concentration, the mobility continues to decrease to $0.9 \times 10^{-4} \mathrm{~cm}^{2} \mathrm{~V}^{-1} \mathrm{~s}^{-1}$ at $5 \%$ DPSQ and further to $0.4 \times 10^{-4} \mathrm{~cm}^{2} \mathrm{~V}^{-1} \mathrm{~s}^{-1}$ at $7.5 \%$ DPSQ. This mobility decrease upon increasing concentration is consistent with the observed trend in fill factor, leading to deterioration in device performance.

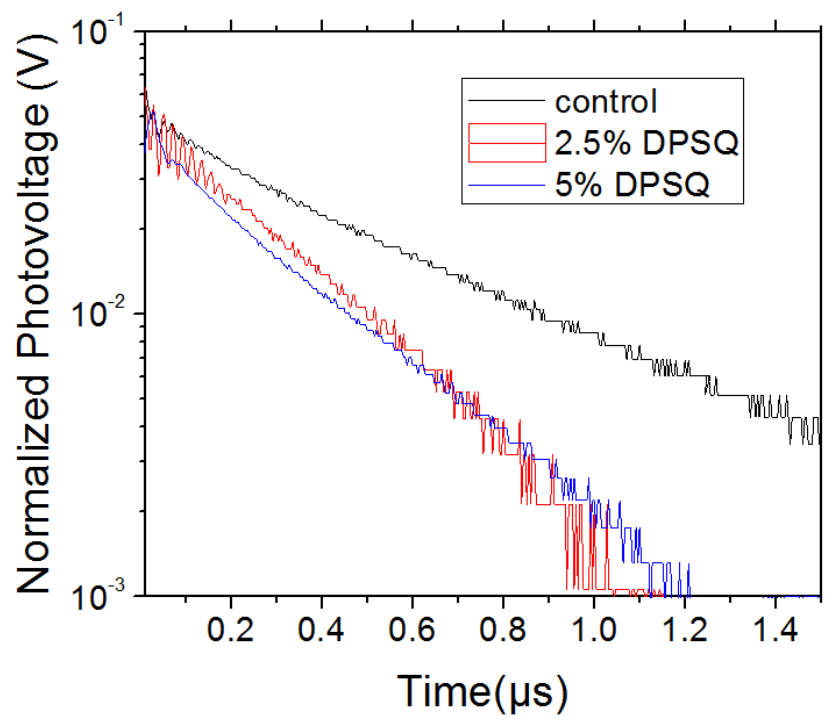

Fig. 5. Transient photovoltage measurements of devices with varying DPSQ concentrations. 
To further understand the decrease of mobility and device performance with large fractions of DPSQ, samples were examined under optical and atomic force microscopes. These samples were prepared by spin-coating the active layer (PCDTBT:PC 71 BM:DPSQ) directly onto glass substrates. The optical micrographs of the samples with varying DPSQ concentrations are depicted in Figure 6 with the insets showing the corresponding AFM images, all with height scales from 0 to $5 \mathrm{~nm}$. In the control device, no significant features were observed in the optical micrograph or AFM image. At a concentration of $2.5 \%$ DPSQ, an aggregate of about $20 \mu \mathrm{m}$ in size was observed in the optical micrograph, while the AFM image of the surrounding matrix shows no signs of aggregation at the nanoscale. More large aggregates were visible in the optical micrograph as the concentration of DPSQ increased further but the AFM image again shows that no aggregation occurs at the nanoscale. This large scale aggregation at high concentrations of DPSQ was a result of the limited solubility of DPSQ in the PCDTBT/PC 71 BM blend. Since these aggregates are likely regions of nearly pristine DPSQ, they are effectively not active in the photovoltaic performance of the devices. Additionally, carriers that enter these domains will not be able to be efficiently extracted. Further, the decrease in electron mobility suggests that there is micro-scale aggregation or incorporation of the dye in the polymer-fullerene matrix interfering carrier extraction. Because of the lack of contrast to distinguish the dye from the rest of the film, any possible aggregation is difficult to reveal by AFM. We conclude that this combination of decreasing lifetime, decreasing mobility, and micro-scale aggregation is the cause of the significant reduction in fill factor observed at high concentrations of DPSQ. This also points to a 
route for a potentially significant improvement in dye doped blend devices by improving the solubility of the dye sensitizer.
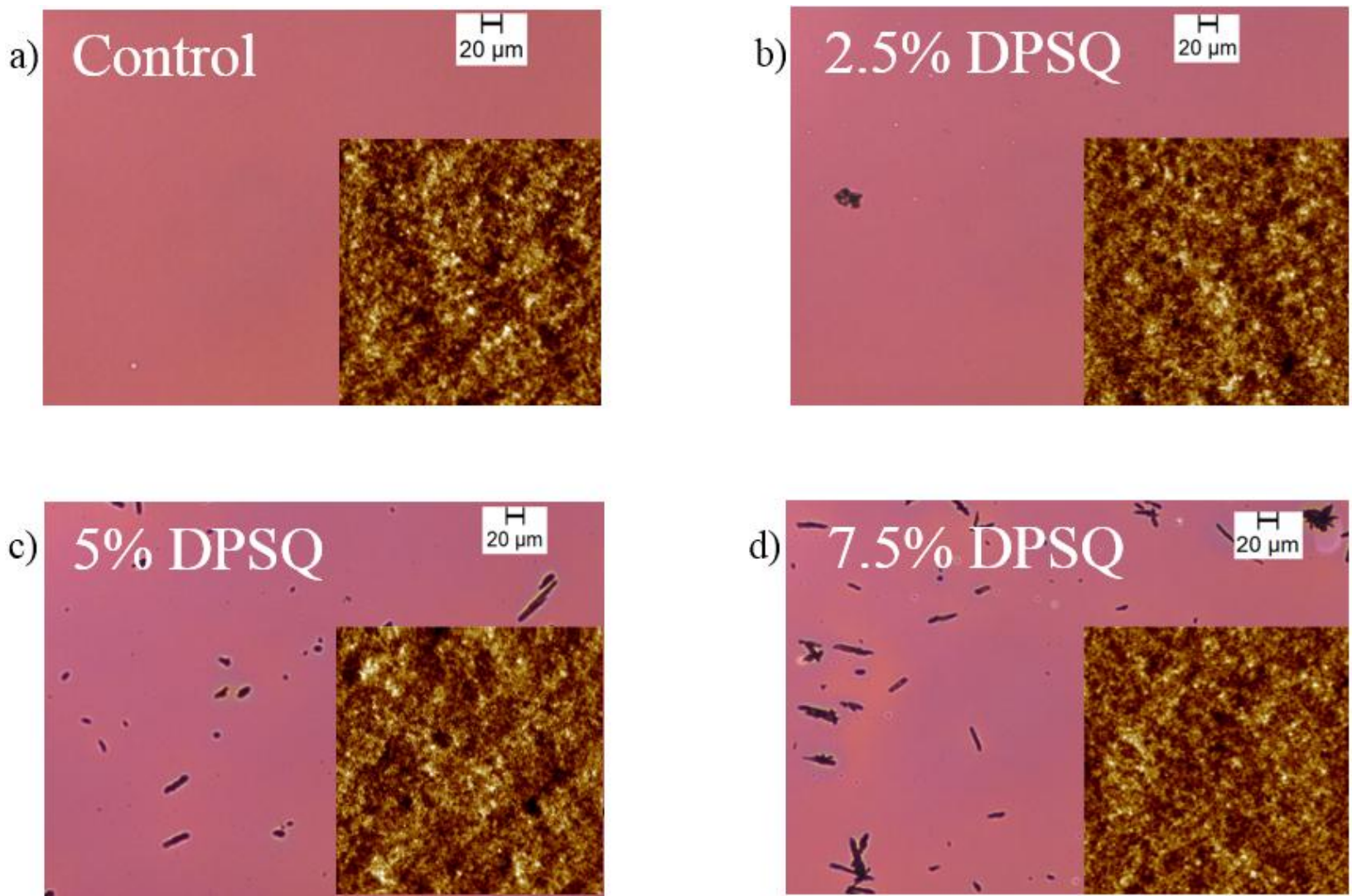

Fig. 6. Optical micrographs of PCDTBT:PC 71 BM:DPSQ layers spin-coated onto glass with a DPSQ concentration of a) $0 \%$, b) $2.5 \%$, c) $5 \%$, d) $7.5 \%$. Inset shows AFM of corresponding samples.

\section{Conclusion}

In summary, we demonstrated that through careful selection of a dye sensitizer, a significant increase in device performance of PSCs is possible. The squaraine dye selected for this work, DPSQ, demonstrated FRET with the commonly studied polymer PCDTBT via photoluminescence studies. By incorporating DPSQ into the active layer of otherwise typical, 
normal structure PCDTBT devices, we were able to achieve significant increases in the PCE. As the concentration increased beyond $2.5 \%$, we saw a decrease in fill factor that ultimately led to a decreased PCE. We found that the decrease in fill factor was a result of decreasing carrier lifetime along with a decrease in carrier mobility combined with aggregation of DPSQ. The utilization of a FRET based dye in PCDTBT helps point to the idea that this is a general strategy which is applicable to a large range of polymer systems. The discovery that aggregation is the limiting factor in dye loading points to the potential for even a greater improvement of device performance through increasing the solubility of the dye molecules used.

Acknowledgements:

The authors would like to acknowledge the support of Office of Naval Research (Award \# N00014-14-1-0173). 


\section{References}

[1] F.C. Krebs, S.A. Gevorgyan, J. Alstrup, A roll-to-roll process to flexible polymer solar cells: model studies, manufacture and operational stability studies, J. Mater. Chem., 19 (2009) 5442. [2] C.E. Small, S. Chen, J. Subbiah, C.M. Amb, S.-W. Tsang, T.-H. Lai, J.R. Reynolds, F. So, High-efficiency inverted dithienogermole-thienopyrrolodione-based polymer solar cells, Nat. Photonics, 6 (2011) 115-120.

[3] L. Lu, T. Xu, W. Chen, E.S. Landry, L. Yu, Ternary blend polymer solar cells with enhanced power conversion efficiency, Nat. Photonics, 8 (2014) 716-722.

[4] J. Liu, Q. Liang, H. Wang, M. Li, Y. Han, Z. Xie, L. Wang, Improving the morphology of PCDTBT:PC70BM Bulk heterojunction by mixed-solvent vapor-assisted imprinting: inhibiting intercalation, optimizing vertical phase separation, and enhancing photon absorption, J. Phys. Chem. C, 118 (2014) 4585-4595.

[5] S.H. Park, A. Roy, S. Beaupré, S. Cho, N. Coates, J.S. Moon, D. Moses, M. Leclerc, K. Lee, A.J. Heeger, Bulk heterojunction solar cells with internal quantum efficiency approaching $100 \%$, Nat. Photonics, 3 (2009) 297-302.

[6] H.J. Son, L. Lu, W. Chen, T. Xu, T. Zheng, B. Carsten, J. Strzalka, S.B. Darling, L.X. Chen, L. Yu, Synthesis and photovoltaic effect in dithieno[2,3-d:2',3'-d']benzo[1,2-b:4,5-

b']dithiophene-based conjugated polymers, Adv. Mater., 25 (2013) 838-843.

[7] S.C. Price, A.C. Stuart, L. Yang, H. Zhou, W. You, Fluorine substituted conjugated polymer of medium band gap yields $7 \%$ efficiency in polymer-fullerene solar cells, J. Am. Chem. Soc., 133 (2011) 4625-4631.

[8] T.Y. Chu, J. Lu, S. Beaupre, Y. Zhang, J.R. Pouliot, S. Wakim, J. Zhou, M. Leclerc, Z. Li, J. Ding, Y. Tao, Bulk heterojunction solar cells using thieno[3,4-c]pyrrole-4,6-dione and dithieno[3,2-b:2',3'-d]silole copolymer with a power conversion efficiency of $7.3 \%$, J. Am. Chem. Soc., 133 (2011) 4250-4253.

[9] C.-Y. Chang, W.-K. Huang, Y.-C. Chang, K.-T. Lee, H.-Y. Siao, High-performance flexible tandem polymer solar cell employing a novel cross-linked conductive fullerene as an electron transport layer, Chem. Mater., 27 (2015) 1869-1875.

[10] L. Dou, J. You, J. Yang, C.-C. Chen, Y. He, S. Murase, T. Moriarty, K. Emery, G. Li, Y. Yang, Tandem polymer solar cells featuring a spectrally matched low-bandgap polymer, Nat. Photonics, 6 (2012) 180-185.

[11] J.Y. Kim, K. Lee, N.E. Coates, D. Moses, T.-Q. Nguyen, M. Dante, A.J. Heeger, Efficient tandem polymer solar cells fabricated by all-solution processing, Science, 317 (2007) 222-225. [12] Z. Hu, S. Tang, A. Ahlvers, S.I. Khondaker, A.J. Gesquiere, Near-infrared photoresponse sensitization of solvent additive processed poly(3-hexylthiophene)/fullerene solar cells by a low band gap polymer, Appl. Phys. Lett., 101 (2012) 053308.

[13] F. Machui, S. Rathgeber, N. Li, T. Ameri, C.J. Brabec, Influence of a ternary donor material on the morphology of a P3HT:PCBM blend for organic photovoltaic devices, J. Mater. Chem., 22 (2012) 15570. 
[14] W. Belcher, K. Wagner, P. Dastoor, The effect of porphyrin inclusion on the spectral response of ternary P3HT:porphyrin:PCBM bulk heterojunction solar cells, Sol. Energ. Mat. Sol. Cells, 91 (2007) 447-452.

[15] J.-S. Huang, T. Goh, X. Li, M.Y. Sfeir, E.A. Bielinski, S. Tomasulo, M.L. Lee, N. Hazari, A.D. Taylor, Polymer bulk heterojunction solar cells employing Förster resonance energy transfer, Nat. Photonics, 7 (2013) 479-485.

[16] G. Chen, H. Sasabe, X.-F. Wang, Z. Hong, J. Kido, A squaraine dye as molecular sensitizer for increasing light harvesting in polymer solar cells, Synth. Met., 192 (2014) 10-14.

[17] H. Xu, H. Ohkita, T. Hirata, H. Benten, S. Ito, Near-IR dye sensitization of polymer blend solar cells, Polymer, 55 (2014) 2856-2860.

[18] Q. An, F. Zhang, L. Li, J. Wang, J. Zhang, L. Zhou, W. Tang, Improved efficiency of bulk heterojunction polymer solar cells by doping low-bandgap small molecules, ACS Appl. Mater. Interfaces, 6 (2014) 6537-6544.

[19] B. Lim, J.T. Bloking, A. Ponec, M.D. McGehee, A. Sellinger, Ternary bulk heterojunction solar cells: addition of soluble NIR dyes for photocurrent generation beyond $800 \mathrm{~nm}$, ACS Appl. Mater. Interfaces, 6 (2014) 6905-6913.

[20] H. Sasabe, T. Igrashi, Y. Sasaki, G. Chen, Z. Hong, J. Kido, Soluble squaraine derivatives for 4.9\% efficient organic photovoltaic cells, RSC Adv., 4 (2014) 42804-42807.

[21] D. Yang, L. Yang, Y. Huang, Y. Jiao, T. Igarashi, Y. Chen, Z. Lu, X. Pu, H. Sasabe, J. Kido, Asymmetrical squaraines bearing fluorine-substituted indoline moieties for highperformance solution-processed small-molecule organic solar cells, ACS Appl. Mater. Interfaces, 7 (2015) 13675-13684.

[22] Q. An, F. Zhang, Q. Sun, J. Wang, L. Li, J. Zhang, W. Tang, Z. Deng, Efficient small molecular ternary solar cells by synergistically optimized photon harvesting and phase separation, J. Mater. Chem. A, 3 (2015) 16653-16662.

[23] S. Paek, H. Choi, H. Jo, K. Lee, K. Song, S.A. Siddiqui, G.D. Sharma, J. Ko, A new unsymmetrical near-IR small molecule with squaraine chromophore for solution processed bulk heterojunction solar cells, J. Mater. Chem. C, 3 (2015) 7029-7037.

[24] H. Xu, T. Wada, H. Ohkita, H. Benten, S. Ito, Molecular design of near-IR dyes with different surface energy for selective loading to the heterojunction in blend films, Sci. Rep., 5 (2015) 9321.

[25] R.M. Clegg, Förster resonance energy transfer-FRET what is it, why do it, and how it's done, in: T.W.J. Gadella (Ed.) Laboratory Techniques in Biochemistry and Molecular Biology, Elsevier, 2009, pp. 1-57.

[26] K. Cnops, B.P. Rand, D. Cheyns, B. Verreet, M.A. Empl, P. Heremans, 8.4\% efficient fullerene-free organic solar cells exploiting long-range exciton energy transfer, Nat. Commun., 5 (2014) 3406.

[27] V. Gupta, V. Bharti, M. Kumar, S. Chand, A.J. Heeger, Polymer-polymer Forster resonance energy transfer significantly boosts the power conversion efficiency of bulk-heterojunction solar cells, Adv. Mater., (2015).

[28] G. Wei, X. Xiao, S. Wang, K. Sun, K.J. Bergemann, M.E. Thompson, S.R. Forrest, Functionalized squaraine donors for nanocrystalline organic photovoltaics, ACS Nano, 6 (2011) 972-978.

[29] V. Adamovich, J. Brooks, A. Tamayo, A.M. Alexander, P.I. Djurovich, B.W. D'Andrade, C. Adachi, S.R. Forrest, M.E. Thompson, High efficiency single dopant white electrophosphorescent light emitting diodes, New J. Chem., 26 (2002) 1171-1178. 
[30] R.A. Street, D. Davies, P.P. Khlyabich, B. Burkhart, B.C. Thompson, Origin of the tunable open-circuit voltage in ternary blend bulk heterojunction organic solar cells, J. Am. Chem. Soc., 135 (2013) 986-989. 